\title{
RIPK3 promotes kidney fibrosis via AKT-dependent ATP citrate lyase
}

\author{
Mitsuru Imamura, ${ }^{1}$ Jong-Seok Moon, ${ }^{1,2}$ Kuei-Pin Chung, ${ }^{1}$ Kiichi Nakahira, ${ }^{1}$ \\ Thangamani Muthukumar, ${ }^{3,4}$ Roman Shingarev, ${ }^{5}$ Stefan W. Ryter, ${ }^{1}$ Augustine M.K. Choi, ${ }^{1,6}$ \\ and Mary E. Choi ${ }^{3,6}$
}

'Division of Pulmonary and Critical Care Medicine, Joan and Sanford I. Weill Department of Medicine, Weill Cornell Medical College, New York, New York, USA. ${ }^{2}$ Soonchunhyang Institute of Medi-bio Science, Soonchunhyang University, Cheonan-si, Chungcheongnam-do, Republic of Korea. ${ }^{3}$ Division of Nephrology and Hypertension, Joan and Sanford I. Weill Department of Medicine, Weill Cornell Medical College, New York, New York, USA. ${ }^{4}$ Department of Transplantation Medicine, New York Presbyterian Hospital-Weill Cornell Medical Center, New York, New York, USA. ${ }^{5}$ Renal Service, Department of Medicine, Memorial Sloan Kettering Cancer Center, New York, New York, USA. ${ }^{6}$ Department of Medicine, New York Presbyterian Hospital-Weill Cornell Medical Center, New York, New York, USA.

Renal fibrosis is a common pathogenic response to injury in chronic kidney disease (CKD). The receptor-interacting protein kinase-3 (RIPK3), a regulator of necroptosis, has been implicated in disease pathogenesis. In mice subjected to unilateral ureteral obstruction-induced (UUO-induced) or adenine diet-induced (AD-induced) renal fibrosis, models of progressive kidney fibrosis, we demonstrate increased kidney expression of RIPK3. Mice genetically deficient in RIPK3 displayed decreased kidney fibrosis and improved kidney function relative to WT mice when challenged with UUO or AD. In contrast, mice genetically deficient in mixed-lineage kinase domain-like protein (MLKL), a downstream RIPK3 target, were not protected from UUO-induced kidney fibrosis. We demonstrate a pathway by which RIPK3 promotes fibrogenesis through the AKT-dependent activation of ATP citrate lyase (ACL). Genetic or chemical inhibition of RIPK3 suppressed the phosphorylation of AKT and ACL in response to TCF- $\beta 1$ in fibroblasts. Inhibition of AKT or ACL suppressed TCF- $\beta 1$-dependent extracellular matrix production and myofibroblast differentiation in fibroblasts. Pharmacological inhibition of ACL suppressed UUO-induced kidney fibrosis. RIPK3 expression was highly regulated in human CKD kidney. In conclusion, we identify a pathway by which RIPK3 promotes kidney fibrosis independently of MLKL-dependent necroptosis as a promising therapeutic target in CKD.

Conflict of interest: The authors have declared that no conflict of interest exists.

Submitted: May 8, 2017

Accepted: December 27, 2017

Published: February 8, 2018

Reference information:

JCI Insight. 2018;3(3):e94979. https:// doi.org/10.1172/jici.insight.94979.

\section{Introduction}

Renal fibrosis is characterized by the excessive production and accumulation of extracellular matrix (ECM) proteins, such as collagen and fibronectin, within the kidney, which strongly correlate with deterioration of kidney function $(1,2)$. Potent profibrotic effects of TGF- $\beta 1$ have been well established (3-7); however, anti-TGF- $\beta$ therapy approaches have fallen short of expectations in human fibrotic diseases $(8,9)$ Hence, there is a critical need for a better understanding of TGF- $\beta 1$ actions and downstream targets.

TGF- $\beta 1$ can activate the serine/threonine kinase AKT by inducing its phosphorylation in fibroblasts (10). AKT regulates many cellular processes, including cell survival, proliferation, and metabolism (11, 12). AKT phosphorylation has been shown to be increased in mouse kidney after unilateral ureteral obstruction (UUO), an established model of progressive renal fibrosis (13). AKT regulates a number of target proteins, including ATP citrate lyase (ACL), a key metabolic enzyme responsible for the synthesis of acetyl-CoA $(14,15)$. Acetyl-CoA is an important metabolic precursor molecule that is utilized for the synthesis of long-chain fatty acids (FAs) and for other metabolic pathways. Our previous studies have uncovered relationships between metabolic dysfunction (including disruption of the glycolytic and FA synthesis pathways) and the regulation of inflammation in organ injury models $(16,17)$. However, few studies to date have addressed the role of metabolic dysfunction in fibrogenesis (18). A recent study has demonstrated alteration in FA metabolism in the kidneys of UUO-treated mice (19). Furthermore, lipid accumulation and defective FA oxidation were observed in tubular epithelial cells in chronic kidney disease (CKD) kidneys and in experimental folic acid-induced kidney fibrosis 
(20). Previously, we have also shown that mitochondrial and metabolic dysfunction can regulate the necroptosis pathway in pulmonary disease (21). Necroptosis, a genetically programmed form of cell death regulated by receptor-interacting protein kinase-1 (RIPK1), RIPK3, and mixed-lineage kinase domain-like protein (MLKL) has emerging significance in human disease (22-26). Few studies have implicated RIPK3 in experimental models of tissue injury, including acute kidney injury and nonalcoholic steatohepatitis (27-31). To date, the role of RIPK3 in the pathogenesis of kidney fibrosis remains largely unknown.

In the current study, we demonstrate a profibrotic role of RIPK 3 using two independent mouse models of kidney fibrosis induced by UUO or adenine diet (AD). We also identify AKT as a downstream signaling target of RIPK3 in kidney fibroblasts. Using the UUO model, we describe a pathway by which RIPK3 promotes kidney fibrogenesis and ECM deposition via the AKT-dependent modulation of the metabolic enzyme ACL. Taken together, our results provide evidence of a RIPK3-dependent, MLKL-independent signaling pathway responsible for kidney fibrogenesis. Furthermore, we identify the metabolic enzyme ACL as a therapeutic target in renal fibrosis.

\section{Results}

RIPK3 promotes kidney fibrogenesis. We first examined the expression of RIPK3 in the UUO model of kidney fibrosis. The kidneys of C57BL/6 mice subjected to UUO displayed higher mRNA expression of Ripk3 (Figure 1A) compared with mice subjected to sham surgery. Increased protein expression of RIPK3 was confirmed at day 7 of UUO by Western blotting (Figure 1B).

To examine the importance of RIPK3 in fibrogenesis, we subjected RIPK3-deficient (Ripk $3^{-/-}$) (C57BL/6 background) and WT mice to UUO or sham surgery. Type-I collagen (Col-1), fibronectin (FN), and $\alpha$-smooth muscle actin ( $\alpha$-SMA), markers of ECM deposition and myofibroblast formation, were increased in the kidney by UUO at day 7 in WT mice, whereas these effects were suppressed in Ripk $3^{-1}$ mice (Figure 1C). Consistently, Colla1 and Acta2 mRNA (encoding Col-1 and $\alpha$-SMA, respectively) expres-

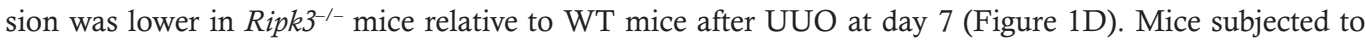
UUO displayed increased collagen deposition in kidney tissue by Masson's trichrome staining compared

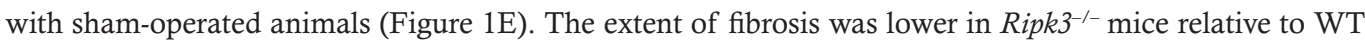
mice subjected to UUO (Figure 1E). Similar to results observed at day 7, Ripk $3^{-1-}$ mice displayed reduced Col-1 and FN expression in the kidney relative to WT mice 13 days after UUO (Supplemental Figure 1; supplemental material available online with this article; https://doi.org/10.1172/jci.insight.94979DS1). These results indicate that Ripk $^{-1-}$ mice are protected against UUO-induced kidney fibrosis.

Immunohistochemical analysis revealed sparse expression of RIPK3 in the interstitial area and no expression in the tubular epithelial cells and glomeruli in kidneys from sham-operated WT mice (Figure $1 \mathrm{~F}$ ). In contrast, kidneys from WT mice subjected to UUO displayed intense interstitial staining of RIPK3 compared with sham kidneys (Figure 1F). RIPK3 expression was also evident in tubular epithelial cells of mice subjected to UUO (Figure 1F). Immunofluorescence staining revealed colocalization of RIPK3 expression with vimentin-positive cells (Figure 1G and Supplemental Figure 2), suggesting that RIPK3 is expressed in fibroblasts. As expected, RIPK3 staining was absent in kidneys from Ripk3-1mice (Figure 1, F and G).

We also performed the TUNEL assay to evaluate the potential role of RIPK3 in cell death in the UUO model. Increased numbers of TUNEL-positive cells were detected in the kidney after UUO at day 7 in WT mice compared with mice subjected to sham surgery. However, the absolute number of TUNEL-positive cells in the kidney tissue of UUO-treated mice was low. In contrast, Ripk $3^{-1-}$ mice displayed fewer TUNEL-positive cells after UUO than WT mice (Figure 1H).

To further confirm the role of RIPK3 in kidney fibrosis, we implemented a model of AD-induced kidney fibrosis, as an alternate model of CKD, which permits assessment of renal function (32). WT mice subjected to AD displayed impaired renal function, as determined by elevated serum creatinine and blood urea nitrogen at day 7 (Supplemental Figure 3) and day 14 (Figure 2A). In contrast, Ripk3 ${ }^{-1-}$ mice displayed improved renal function relative to WT mice after AD at day 7 (Supplemental Figure 3) and day 14 (Figure 2A). Protein expression of RIPK3 was higher in the kidneys of WT mice at day 14 of AD relative to mice on the control diet (Figure 2B). Col-1, FN, and $\alpha$-SMA expression levels were higher in the kidney at day 14 after $\mathrm{AD}$ in WT mice, whereas the expression of these proteins was lower in Ripk $3^{-1-}$ mice (Figure 2B). Mice subjected to AD displayed increased collagen deposition in kidney tissue, as indicated by Masson's trichrome staining, compared with animals on the control diet (Figure 2C). The extent of fibrosis was lower in Ripk $3^{-1}$ 

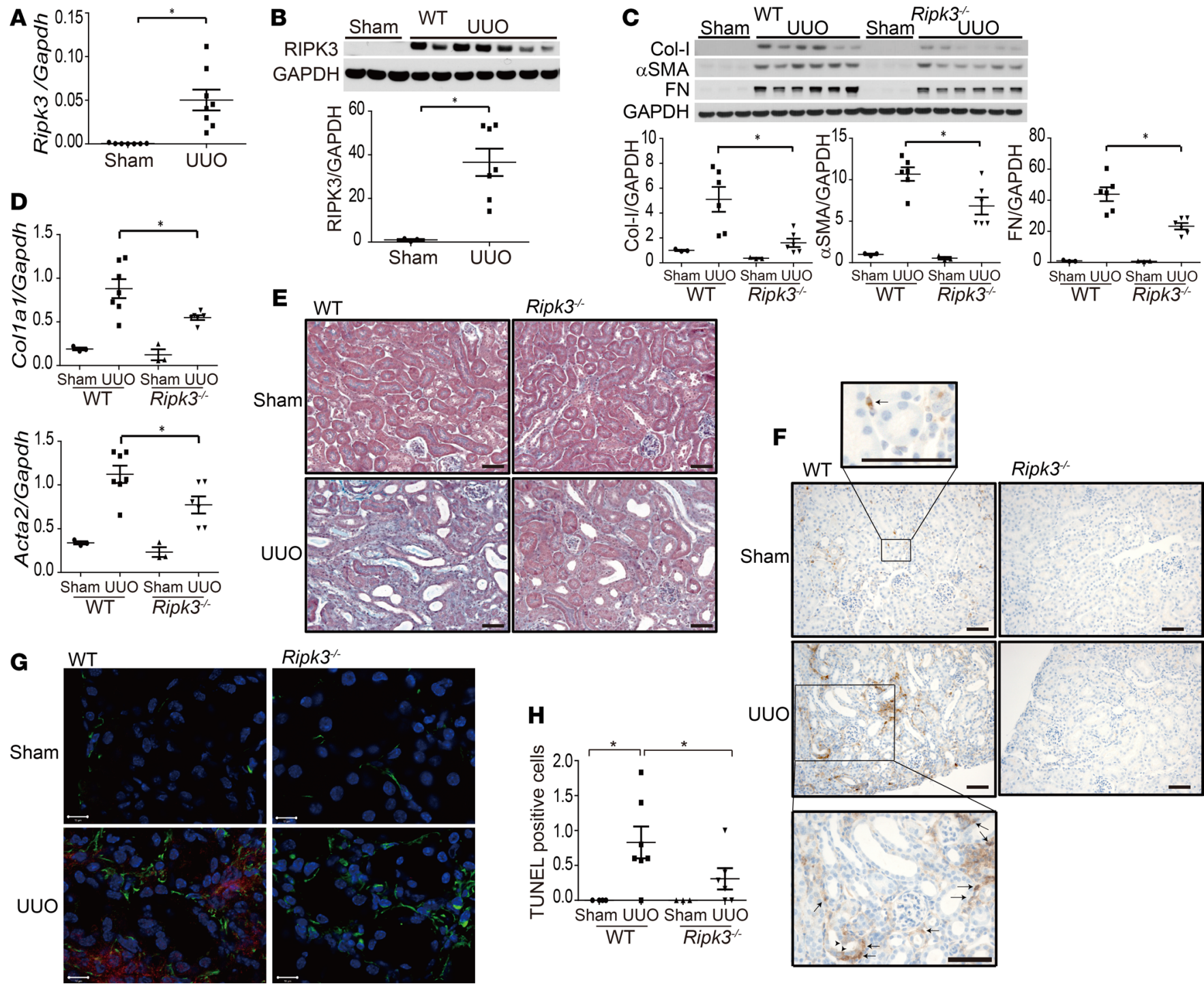

Figure 1. RIPIK3 promotes kidney fibrosis in the UUO model. WT (A-H) or Ripk3 ${ }^{-1-}$ mice $(\mathbf{C}-\mathbf{H})$ were subjected to sham operation or UUO, and kidneys were harvested at 7 days after surgery. (A) Ripk3 mRNA expression was analyzed by qRT-PCR. Gapdh was the standard $(n=7$, WT Sham; $n=8$, WT UUO). * $P<$ 0.05 by Mann-Whitney test. (B) RIPK3 expression was determined by Western blot analysis. GAPDH was the standard ( $n=3$, WT Sham; $n=7$, WT UUO). Dot plots represent densitometric analysis of Western blot data (mean \pm SEM, 3 independent experiments). ${ }^{*} P<0.05$ by Mann-Whitney test. (C) Kidney tissue lysates were subjected to Western blot analysis for $\alpha$-smooth muscle actin ( $\alpha$ SMA), type-I collagen (Col-I), and fibronectin (FN). GAPDH was the standard. Dot plots represent densitometric analysis of Western blot data (mean \pm SEM, 3 independent experiments). ${ }^{*} P<0.05$ compared with WT UUO mice by ANOVA with Newman-Keuls post-hoc test. (D) Kidney tissues were analyzed by qRT-PCR for Col1a1 and Acta2 mRNA expression. Gapdh was the standard. ${ }^{*} P<0.05$ compared with WT UUO mice by ANOVA with Newman-Keuls post-hoc test (mean \pm SEM, 3 independent experiments). (E and F) Kidney tissues were stained with (E) Masson's trichrome or (F) immunostained for RIPK3. Representative images are shown. Tubular epithelial cells (arrowheads) and interstitial cells (arrows) are indicated. Scale bars: $100 \mu \mathrm{m}$. (G) Immunofluorescence staining for RIPK3 (red), vimentin (green), and Hoechst (blue). Scale bars: $10 \mu \mathrm{m}$. C-G; $n=6$ for UUO; $n=3$ for Sham. (H) Quantitative analysis of TUNEL-positive cells. The number of TUNEL-positive cells was counted in 5 random high-power fields (HPF) of the left kidney of the WT mice and Ripk $3^{-1-}$ mice 7 days after UUO or sham surgery. The dot represents the average of the number of TUNEL-positive cells per HPF for each mouse. WT Sham $(n=4)$, WT UUO $(n=7), \operatorname{Ripk}^{-1-} \operatorname{Sham}(n=3), \operatorname{Ripk3^{-/-}}$ UUO $(n=6)$. ${ }^{*} P<$ 0.05 compared with WT UUO mice by ANOVA with Newman-Keuls post-hoc test. All values represent mean \pm SEM from 3 independent experiments.

mice relative to WT mice subjected to AD (Figure 2C). Immunohistochemical analysis of kidneys from WT mice subjected to AD displayed intense staining of RIPK3 in tubular epithelial cells and interstitium (Figure 2D). Analysis of kidney cell death by TUNEL staining failed to detect significant TUNEL-positive cells in

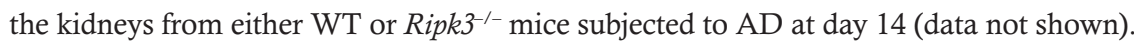

Taken together, these results in two independent mouse models of renal fibrosis suggest that RIPK3 is critically involved in the pathogenesis of kidney fibrosis. To further investigate the mechanisms by which RIPK3 may regulate fibrogenesis, we focused on the UUO model in subsequent experiments. 

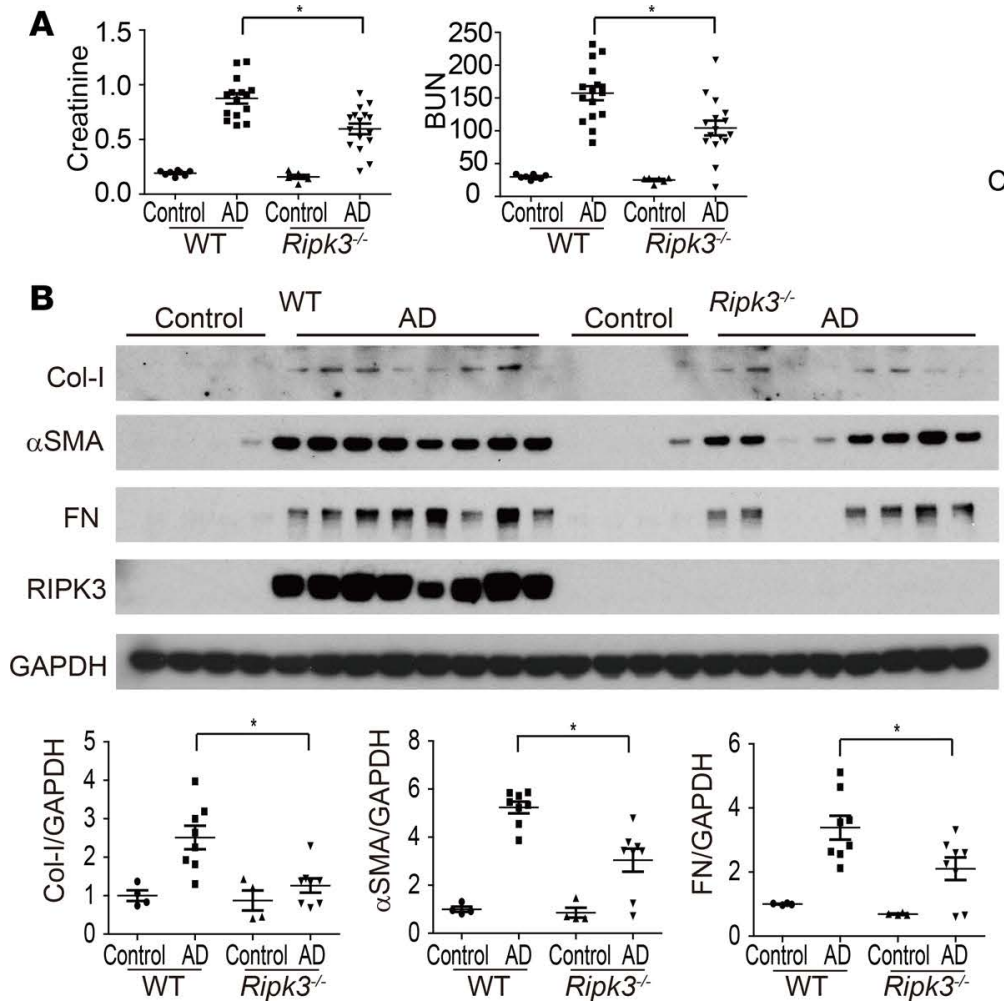

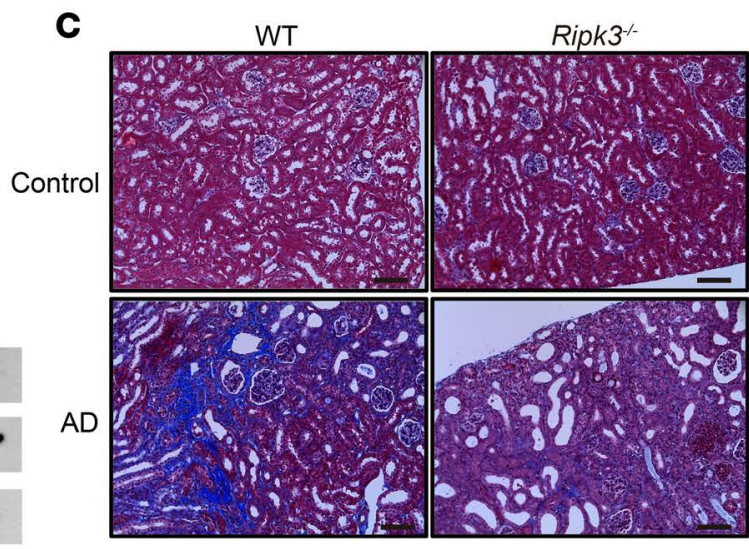

D

WT

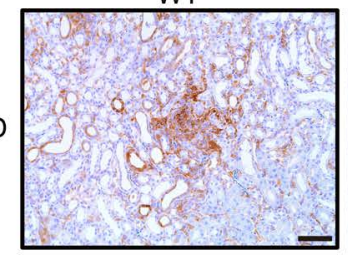

Ripk3-

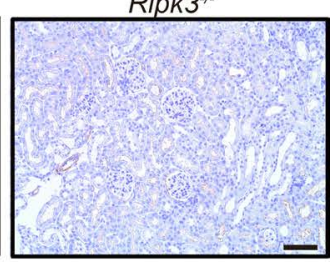

Figure 2. RIPK3 promotes kidney fibrosis in the AD model. WT or Ripk3 $3^{-1-}$ mice were subjected to control or $0.2 \%$ adenine diet (AD), and kidneys and serum were harvested at 14 days. (A) Serum creatinine and BUN were measured (mean \pm SEM). ${ }^{*} P<0.05$ compared with WT AD-fed mice by ANOVA with Newman-Keuls post-hoc test. WT control diet $(n=8)$, WT AD $(n=16)$, Ripk3 $3^{-/-}$control diet $(n=6)$, Ripk $3^{-/-}$AD $(n=16)$. (B) Kidney tissue lysates were subjected to Western blot analysis for type-I collagen (Col-I), $\alpha$-smooth muscle actin ( $\alpha$ SMA), fibronectin (FN), and RIPK3. GAPDH was the standard. Dot plots represent densitometric analysis of Western blot data (mean \pm SEM). ${ }^{*} P<0.05$ compared with WT AD mice by ANOVA with Newman-Keuls post-hoc test. WT control diet $(n=4)$, WT AD $(n=8)$, Ripk3 $3^{-1-}$ control diet $(n=4)$, Ripk3 ${ }^{-/-}$AD $(n=8)$. (C and D) Kidney tissues were stained with (C) Masson's trichrome or (D) immunostained for RIPK3. Representative images are shown. Scale bars: $100 \mu \mathrm{m}$.

MLKL deficiency fails to protect against kidney fibrosis. MLKL is a downstream target of RIPK3, and phospho-MLKL (p-MLKL) is recognized as a specific marker of necroptosis $(22,33)$. However, recent studies suggest that RIPK3 may have pleiotropic effects on cellular processes (34-36), some of which may occur independently of MLKL (37). Furthermore, mice genetically deficient in RIPK3 or MLKL have been shown to exhibit differential phenotypes in acute kidney injury models $(38,39)$.

To determine the role of MLKL in UUO-induced kidney fibrosis, we next subjected $\mathrm{Mlkl}^{\text {-- }^{-}}$and $\mathrm{Mlkl}^{+/+}$ mice to UUO or sham surgery. The kidneys of $\mathrm{Mlkl}^{+/+}$mice subjected to UUO displayed higher expression of MLKL and p-MLKL compared with mice subjected to sham surgery at day 7 (Figure 3A). Col-1, FN, and $\alpha$-SMA were increased in the kidney by UUO at day 7 in both $\mathrm{Mlkl}^{-1}$ and $\mathrm{Mlkl}^{+/+}$mice (Figure 3A). Col1a1, Acta2, and Tgfb1 mRNA expression in the kidney tissue of $M l k t^{-1}$ mice was not different from that of $\mathrm{Mlkl}^{+/+}$mice (Figure 3B). The extent of fibrosis in $\mathrm{Mlkl}^{-1-}$ mice, as determined by Masson's trichrome staining, was similar to that of $\mathrm{Mlkl}^{+/+}$mice subjected to UUO (Figure 3C). These data suggest that MLKL does not play an important role in kidney fibrosis and, furthermore, that RIPK3 promotes kidney fibrosis in an MLKL-independent manner.

RIPK3 is not essential for cytokine responses in UUO. RIPK3 has previously been reported to regulate inflammation and cytokine gene expression in an animal model of colitis (35). Therefore, we evaluated the effect of RIPK3 on the expression of TGF- $\beta 1$ and inflammatory cytokines by qPCR. Tgfb1, Illb, Tnfa, and Il6 mRNA expression was higher in the kidney in response to UUO at day 3 (Supplemental Figure 4) and day 7 (Figure 4A) compared with sham-treated animals. Ripk3 ${ }^{-/-}$mice displayed similar expression of these cytokines relative to WT mice in response to UUO at day 3 (Supplemental Figure 4) and day 7 (Figure 4A). These results suggest that promotion of kidney fibrosis by RIPK3 is not associated with the differential gene regulation of either proinflammatory cytokines or TGF- $\beta 1$ in the UUO model. 


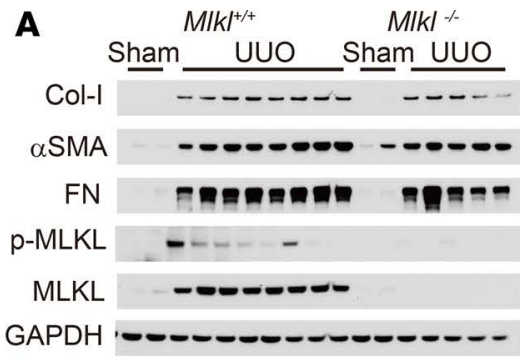

B

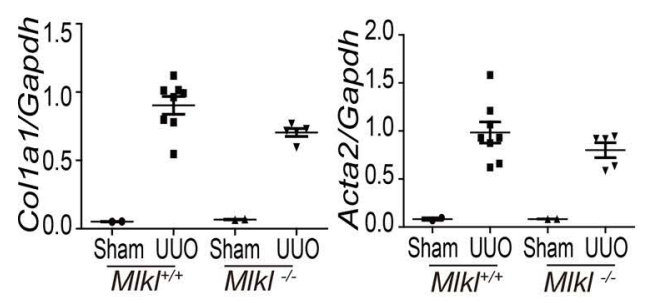

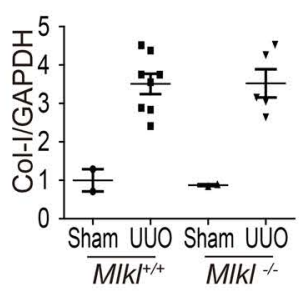

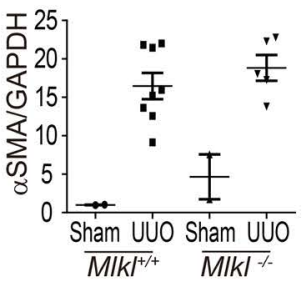

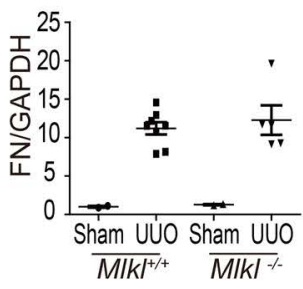

$M \mid k l^{+/+}$
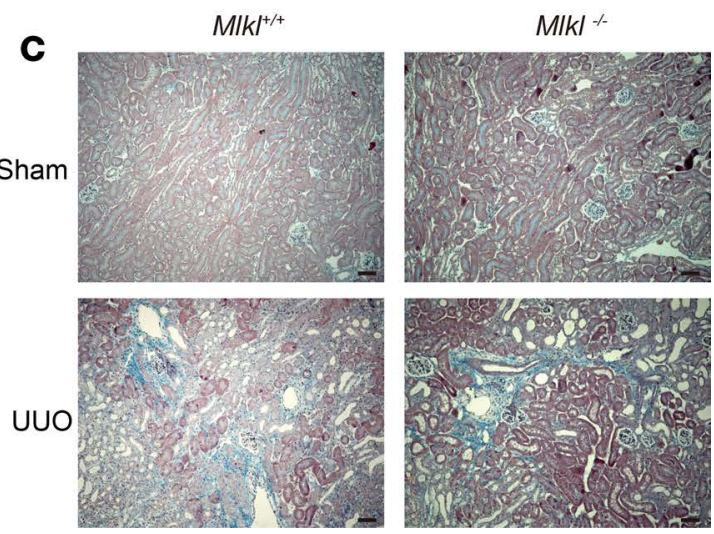

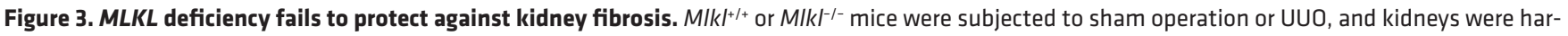
vested at 7 days. (A) Kidney tissue lysates were subjected to Western blot analysis for $\alpha$-smooth muscle actin ( $\alpha$ SMA), type-I collagen (Col-I), fibronectin (FN), MLKL, and p-MLKL. GAPDH was the standard. Dot plots represent densitometric analysis of Western blot data. (B) Kidney tissues were analyzed by qRT-PCR for Col1a1, Acta2, and Tgfb1 mRNA expression. Gapdh was the standard. Values represent mean \pm SEM. Data were analyzed by ANOVA with

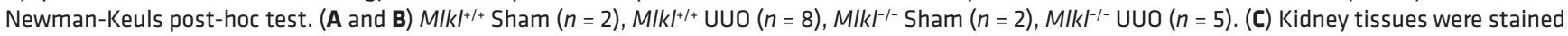
with Masson's trichrome. Representative images are shown. Scale bars: $100 \mu \mathrm{m}$.

RIPK3-dependent AKT and ACL activation are upregulated in kidney fibrosis. Previously, we have reported that metabolic deregulation, including disruption of the glycolytic and FA synthesis pathways, can regulate inflammasome-dependent inflammation in organ injury models $(16,17)$. We therefore sought to determine the importance of the FA synthesis pathway in kidney fibrogenesis by measuring the expression of key enzymes of this pathway in mice subjected to UUO. The expression of phospho-ACL [p-ACL (Ser455)], which generates acetyl-CoA for FA synthesis, was higher in the kidney tissue of WT mice subjected to UUO relative to sham surgery (Figure 4B), whereas FA synthase (FASN), the rate-limiting enzyme in de novo FA synthesis, was unchanged. The expression of phospho-AKT [p-AKT (Ser473)], which regulates ACL, was also higher in response to UUO (Figure 4B). Interestingly, Ripk3 ${ }^{-1-}$ mice displayed lower p-AKT and p-ACL expression in the kidney relative to WT mice after UUO (Figure 4B), indicating that RIPK3 is crucial for regulation of the AKT/ACL pathway during kidney fibrogenesis.

RIPK3 is required for TGF- $\beta 1$-dependent responses in fibrogenesis. Injured epithelial cells produce TGF- $\beta 1$, which induces the proliferation and activation of interstitial fibroblasts (40). On the basis of our observation that TGF- $\beta 1$ expression was not altered by RIPK3 deficiency in UUO (see Figure 4A), we hypothesized that RIPK3 acts downstream of the TGF- $\beta$ pathway in fibroblasts. We therefore tested whether RIPK 3 can be regulated by TGF- $\beta$ in fibroblasts in vitro. TGF- $\beta 1$ induced RIPK3 expression and activated the AKT/ ACL pathway in a dose- and time-dependent manner in NIH 3T3 fibroblasts (Figure 5, A and B). Next, we determined the role of RIPK3 in regulating TGF- $\beta 1$-dependent responses in vitro using genetic interference. Transfection of NIH 3T3 fibroblasts with RIPK3-targeted siRNA reduced TGF- $\beta 1$-dependent phosphorylation of AKT and ACL in NIH 3 T3 fibroblasts (Figure 5C). RIPK3-targeted siRNA also reduced TGF- $\beta 1$ dependent Col-1 and $\alpha$-SMA expression (Figure 5D). Similar results were obtained with the RIPK3 inhibitor compound GSK'872 (41, 42), which reduced the expression of Col-I, $\alpha$-SMA, FN, p-AKT, and p-ACL, in response to TGF- $\beta 1$ stimulation (Figure $5 \mathrm{E}$ ). These data suggest that RIPK3 is required for TGF- $\beta 1$-induced activation of AKT/ACL signaling. Moreover, a necroptosis inhibitor, necrostatin-1 (Nec-1), also reduced the expression of Col-I, $\alpha$-SMA, FN, p-AKT, and p-ACL in response to TGF- $\beta 1$ stimulation (Figure $5 \mathrm{~F}$ ), implying that the necrosome complex may be involved in regulation of this pathway. 


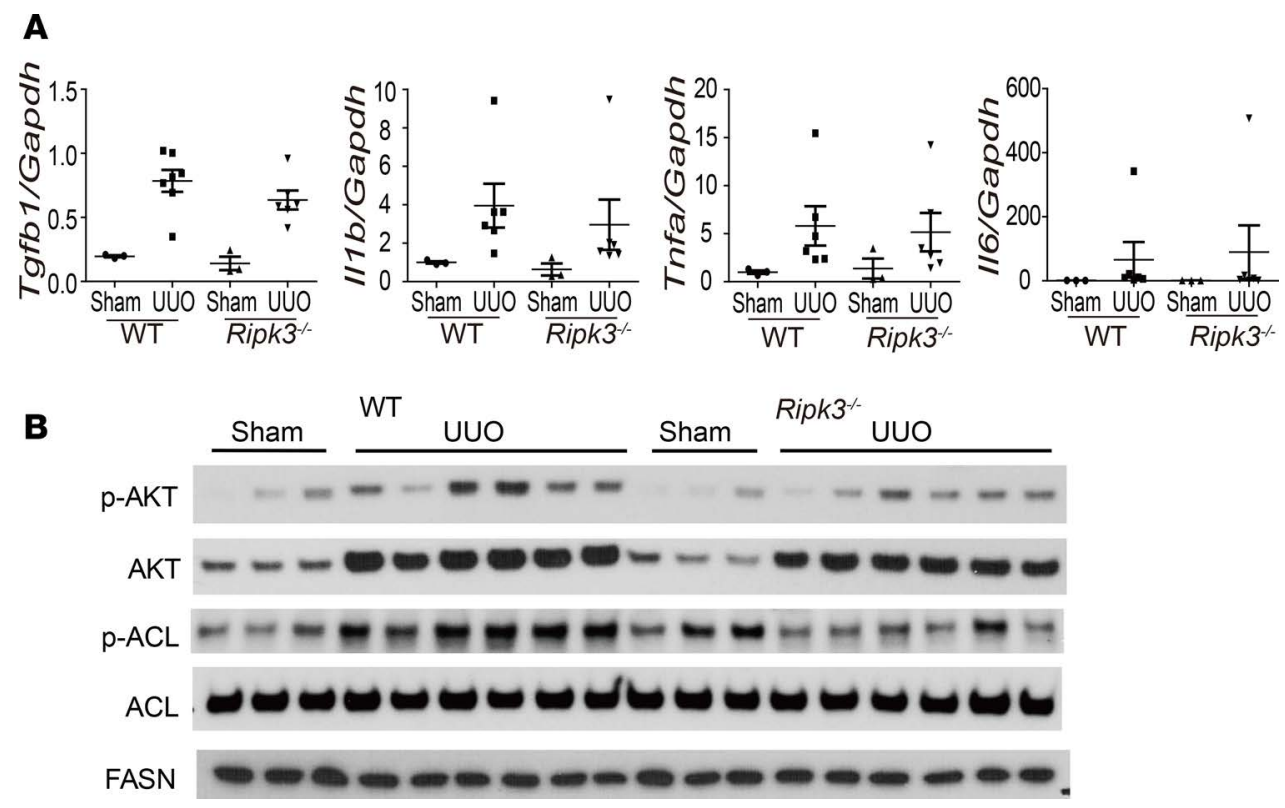

Figure 4. RIPK3 regulates AKT and ACL. WT and Ripk3-/- mice were subjected to UUO or sham surgery, and kidneys were harvested after 7 days. (A) Expression of Tgfb1, I/1b, Tnfa, and II6 mRNA was analyzed by qRT-PCR. Gapdh was the standard ( $n=6$ for UU0; $n=3$ for Sham). Values represent mean \pm SEM, with 3 independent experiments. (B) Kidney tissue was subjected to Western blot analysis of phospho-AKT (Ser473) (p-AKT), AKT, phospho-ATP citrate lyase (Ser455) (p-ACL), total ACL, and fatty acid synthase (FASN) expression.

Genetic and pharmacologic inhibition of AKT and ACL abrogates TGF- $\beta 1$-dependent ECM production. We next used genetic interference and chemical inhibitors of AKT to further confirm a profibrogenic pathway involving AKT-dependent ACL regulation. Transfection of NIH 3T3 fibroblasts (Figure 6A) or primary human kidney fibroblasts (Figure 6B) with AKT1-targeted siRNA reduced TGF- $\beta 1$-dependent Col-1, $\alpha$-SMA, and FN expression and phosphorylation of ACL. The AKT inhibitor compound (AKTi) also reduced the expression of Col-I, $\alpha$-SMA, FN, p-AKT, and p-ACL in response to TGF- $\beta 1$ stimulation in both NIH 3T3 fibroblasts (Figure 6C) and human kidney fibroblasts (Figure 6D). We then used genetic inhibition and chemical inhibitors for ACL to further examine the role of ACL in fibrogenesis. Col-I, $\alpha$-SMA, and FN expression in response to TGF- $\beta 1$ stimulation was also reduced by the ACL-targeted siRNA and ACL inhibitor compound (ACLi) in both NIH 3T3 fibroblasts (Figure 7, A and C) and human kidney fibroblasts (Figure 7, B and D). Moreover, ACLi and AKTi inhibited TGF- $\beta 1$-induced cell proliferation in NIH 3 T3 fibroblasts (Figure 7E). These data suggest that sequential activation of AKT and ACL is critical for ECM production, myofibroblast formation, and proliferation induced by TGF- $\beta 1$ in fibroblasts.

Pharmacological inhibition of ACL reduces UUO-induced kidney fibrosis. We next tested the potential of the AKT/ACL pathway as a therapeutic target in kidney fibrosis. TGF- $\beta 1$ and AKT are upstream signaling regulators with pleiotropic effects in multiple cell types and organs. To date, anti-TGF- $\beta$ therapy approaches have fallen short of expectations in human fibrotic diseases $(8,43)$. AKTi may have multiple targets, such as mTOR signaling $(44,45)$, in addition to inhibiting the ACL pathway. Therefore, we focused on ACL as a potential therapeutic target, downstream of the TGF- $\beta 1$-induced AKT pathway. ACLi compounds have received attention as suppressors of tumor cell growth $(46,47)$. WT mice were injected once with ACLi on day 4 after sham or UUO surgery, and kidney tissues were harvested for analysis on day 7 (Figure 8A). Masson's trichrome staining revealed that collagen deposition was lower in ACLi-treated mice, relative to control mice, after UUO (Figure 8B). ACLi inhibited UUO-dependent expression of Col-I, $\alpha$-SMA, and FN (Figure $8 \mathrm{C}$ ). These results suggest a therapeutic potential of ACL inhibition in kidney fibrosis.

RIPK3 is regulated in human CKD. Finally, we explored the role of RIPK3 in human kidney disease. Kidney allograft biopsy tissues obtained from CKD patients with diabetic nephropathy and histological evidence of tubulointerstitial fibrosis (TIF) demonstrated increased interstitial immunostaining of RIPK3 compared with control kidney biopsy tissues without diabetic nephropathy (Figure 9A). We determined that the relative RIPK3 mRNA expression, as detected by quantitative real-time PCR (qRT-PCR), was higher 
A

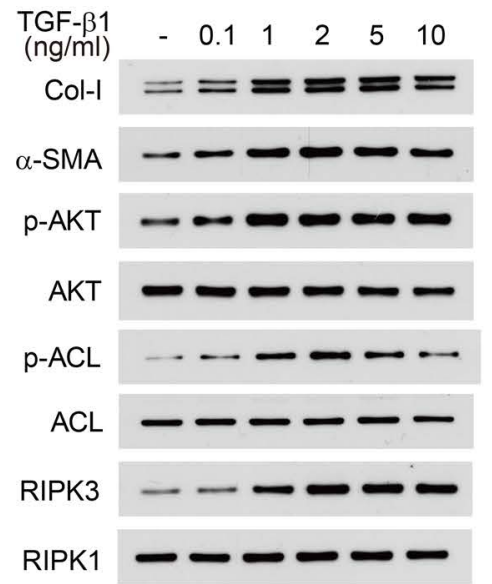

B

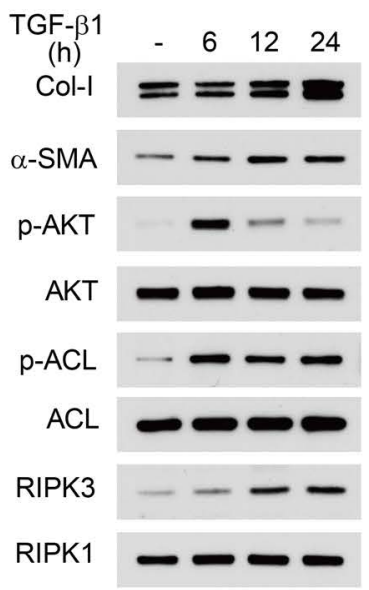

C

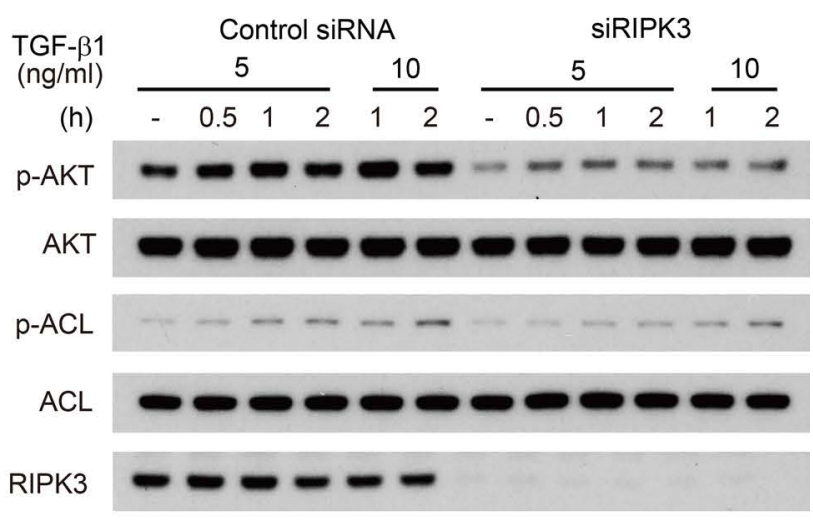

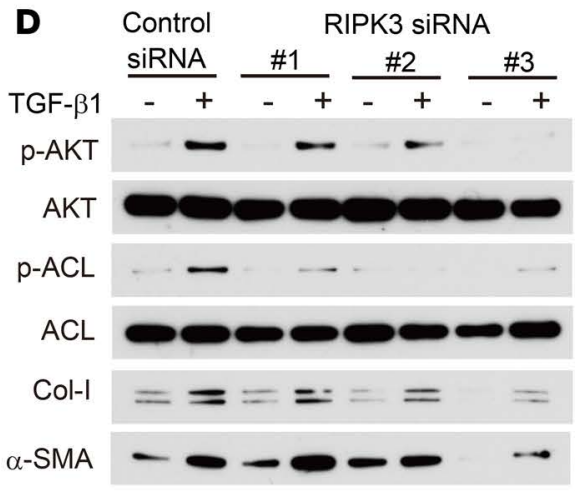

E

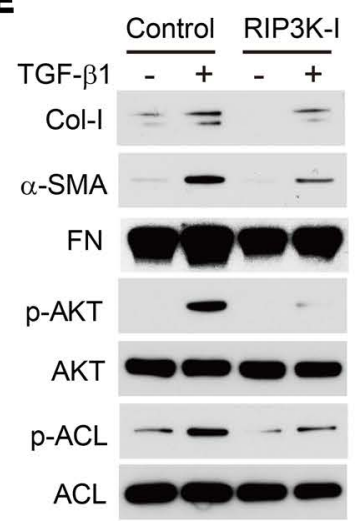

$\mathbf{F}$

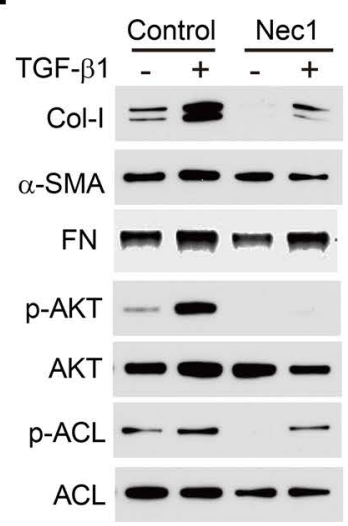

Figure 5. RIPK3 regulates TGF-ק1-induced ECM production via the AKT/ACL pathway in fibroblasts. NIH 3T3 fibroblasts were stimulated with (A) TCF- $\beta 1$ (0.1-10 ng/ml) for 24 hours or (B) TCF- $\beta 1(5 \mathrm{ng} / \mathrm{ml})$ for 6,12 , and 24 hours. Cell lysates were subjected to Western blot analysis for Col-I, $\alpha$-SMA, phospho-AKT (Ser473) (p-AKT), AKT, phospho-ATP citrate lyase (p-ACL), ACL, RIPK3, RIPK1, and $\beta$-actin. (C-F) NIH 3 T3 fibroblasts were treated with (C) nontargeting siRNA (control siRNA) or a mixture of RIPK3 siRNA (SMART Pool) (siRIPK3) for 24 hours and then stimulated with or without TGF- $\beta 1$ $(5 \mathrm{ng} / \mathrm{ml}$, or $10 \mathrm{ng} / \mathrm{ml})$ for 30 minutes to 2 hours. Additionally, NIH 3 T3 fibroblasts were treated with (D) control siRNA or 3 independent siRNA targeting RIPK3 (RIPK3 siRNA \#1, \#2, and \#3) for 6 hours and then stimulated with or without TGF- $\beta 1$ ( $5 \mathrm{ng} / \mathrm{ml})$ for 15 hours. NIH 3 T3 fibroblasts were also treated with (E) RIP3K inhibitor (RIP3K-I) $(10 \mu \mathrm{M})$ or DMSO (Control) for 12 hours and then stimulated with or without TCF- $\beta 1$ (5 ng/ml) for 12 hours. Otherwise, NIH 3 T3 fibroblasts were treated with (F) necrostatin-1 (Nec-1) $(60 \mu \mathrm{M})$ or DMSO (Control) for 1 hour, followed by stimulation with or without TGF- $\beta 1$ ( $5 \mathrm{ng} / \mathrm{ml})$ for 24 hours. Western blot analysis for expression of RIPK3, Col-I, $\alpha$-SMA, FN, p-AKT, AKT, p-ACL, ACL, and $\beta$-actin is shown.

in kidney biopsy samples with TIF compared with control biopsy samples of normal kidney tissue margins from tumor resection (Figure 9B, and Supplemental Table 1). Interestingly the patient sample with the lowest RIPK3 mRNA level in the CKD with TIF group had only minimal evidence of TIF. RIPK3 mRNA was increased in biopsy samples with interstitial fibrosis involving $>5 \%$ of cortical area (Banff fibrosis score ci1-3) compared with interstitial fibrosis involving $\leq 5 \%$ of cortical area (Banff fibrosis score ci0) (Figure 9C). RIPK3 mRNA expression strongly correlated with the interstitial fibrosis score $(P=0.0423, \mathrm{r}=0.5126)$ but not with serum creatinine $(P=0.2681, \mathrm{r}=0.2946)$ (Figure 9D). These results demonstrate that RIPK3 expression is increased in kidney biopsies with interstitial fibrosis, suggesting that RIPK3 may contribute to fibrogenesis in human CKD.

\section{Discussion}

In the current study, we have uncovered that RIPK3 exerts profibrotic effects in the UUO- and AD-induced models of kidney fibrosis in vivo as well as in TGF- $\beta 1$-dependent fibrogenic responses in vitro. Renal expression of RIPK3 was markedly induced in the kidney following either UUO or AD. Moreover, RIPK3 deficiency provided renoprotective effects, as evidenced by reduced levels of ECM in kidneys in response to $\mathrm{UUO}$ or $\mathrm{AD}$, and improved kidney function in response to $\mathrm{AD}$. Our findings establish an important 
A

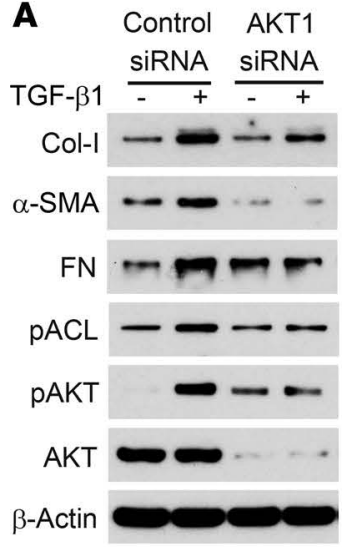

B

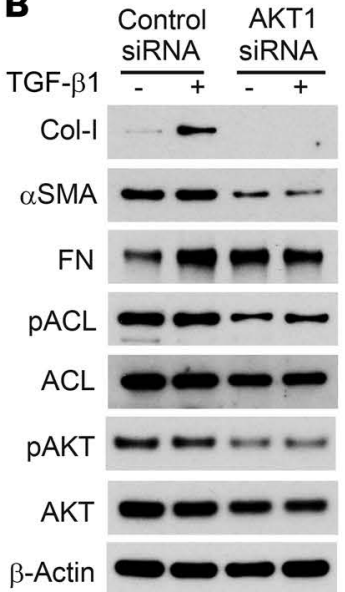

C

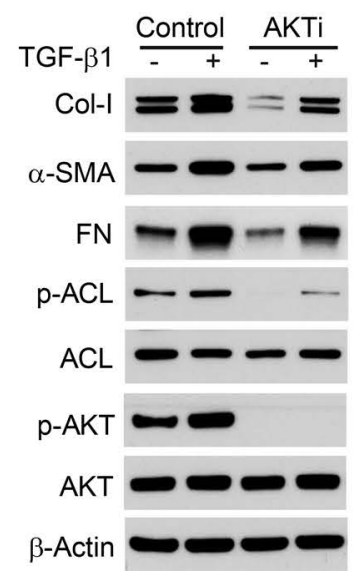

D

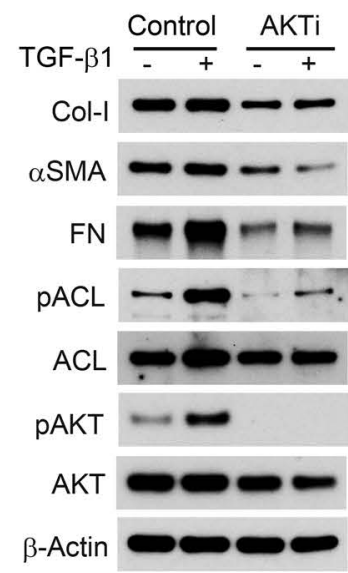

Figure 6. Inhibition of AKT suppresses TGF-ק1-induced ECM production in fibroblasts. (A and B) NIH 3T3 fibroblasts (A) and human kidney fibroblasts (B) were treated with nontargeting siRNA (control siRNA) or AKT1 siRNA for 24 hours and then stimulated with or without TGF- $\beta 1$ ( $5 \mathrm{ng} / \mathrm{ml}$ ) for 24 hours, followed by Western blot analysis as shown. (C and D) NIH 3 T3 fibroblasts (C) and human kidney fibroblasts (D) were treated with $10 \mu \mathrm{M}$ AKT inhibitor (AKTi) or DMSO (Control) and stimulated with or without TCF- $\beta 1(5 \mathrm{ng} / \mathrm{ml})$ for 24 hours, followed by Western blot analysis as shown.

link between kidney fibrogenesis and RIPK3. Furthermore, our studies demonstrate that RIPK3 is highly regulated in human CKD. Taken together, these results suggest the importance of the contribution of the RIPK3-dependent pathway to the pathogenesis of kidney fibrosis and suggest that it is a potential therapeutic target in CKD.

We have observed reduced histological and tissue markers of fibrosis in Ripk $3^{--}$mice relative to WT mice subjected to UUO or AD. In support of our current findings, a recent study has shown that Nec-1, an inhibitor of the RIPK1-dependent necrosome, can suppress kidney fibrosis after UUO (48). We also report here that RIPK3 may promote the phosphorylation of AKT, an event typically associated with cell survival and proliferation, rather than cell death. A previous report showed that RIPK3 can suppress PDGF-induced AKT activation in vascular smooth muscle cells (49). Thus, RIPK3 may have cell-type and inducer-specific effects on AKT signaling. Several studies have implicated the AKT pathway in CKD (50) and kidney fibrosis $(13,51) . A k t 2^{--}$mice displayed reduced kidney fibrosis after UUO relative to WT mice (51). A PI3K inhibitor, which suppresses AKT activation, attenuated kidney fibrosis after UUO (13). These studies support our finding that a RIPK3/AKT pathway may regulate fibrogenesis in obstructed kidneys. Our data show that ACL was phosphorylated in response to TGF- $\beta 1$ and that genetic interference of ACL or treatment with ACLi suppressed TGF- $\beta 1$-induced cell proliferation, myofibroblast formation, and ECM production in NIH $3 \mathrm{~T} 3$ fibroblasts and human kidney fibroblasts. ACLi also attenuated interstitial fibrosis in obstructed kidneys. To our knowledge, this is the first study that identifies a role of ACL in fibrogenesis. Although ACL generates acetyl-CoA used for FA biosynthesis, we cannot exclude the possible involvement of other metabolic pathways dependent on acetyl-CoA.

A limitation of our study is that we were unable to further elucidate the mechanism by which RIPK3 can directly or indirectly promote the phosphorylation of AKT in fibroblasts. We were also unable to demonstrate a direct intermolecular interaction of RIPK3 and AKT (data not shown). Further studies are warranted to clarify this mechanism. A second limitation is that we focused primarily on fibroblasts in our mechanistic studies. We observed that RIPK3 expression was localized in the tubulointerstitial area and also in tubular epithelial cells in the kidneys of mice subjected to either UUO or AD. Thus, we cannot exclude a possible functional role of RIPK3 in other cell types, such as tubular epithelial cells.

RIPK3 forms a "necrosome" complex with RIPK1 and MLKL after TNF- $\alpha$ stimulation to act as a critical regulator of necroptosis (22-26). However, recent studies indicate that RIPK3 can activate signaling pathways independently of MLKL to regulate cellular processes, suggesting that they do not always act in tandem (37). RIPK3-induced cardiomyocyte necroptosis was found to occur independently of RIPK1 and MLKL and required $\mathrm{Ca}^{2+}$-calmodulin-dependent protein kinase, a novel RIPK3 substrate (52). Moreover, in addition to its central role in necroptosis, evidence suggests that RIPK3 may be involved in regulating other cellular processes. For example, a RIPK3-dependent pathway regulates inflammasome activation by RNA virus (34). RIPK3 also 


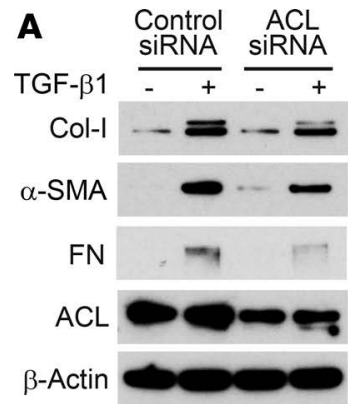

D

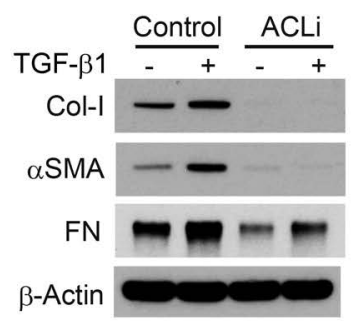

B

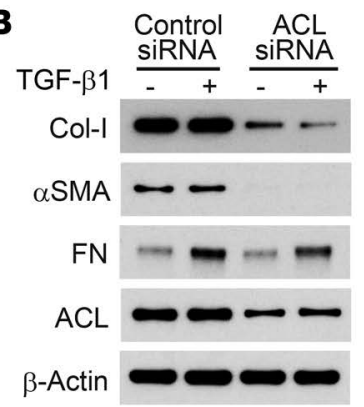

C

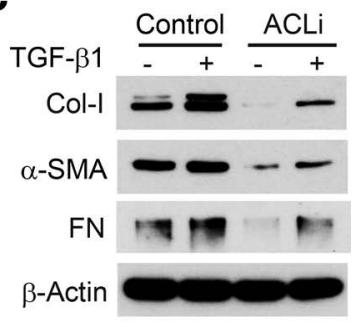

\section{$\mathbf{E}$}

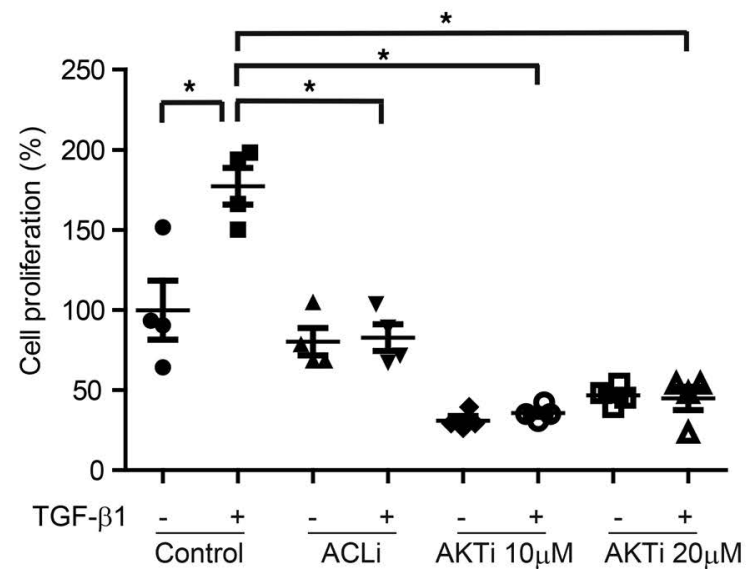

Figure 7. Inhibition of ACL suppresses TGF- $\beta 1$-induced ECM production in fibroblasts. (A and B) NIH 3 T3 fibroblasts (A) and human kidney fibroblasts (B) were stimulated with or without TCF- $\beta 1(5 \mathrm{ng} / \mathrm{ml})$ for 3 hours and treated with control siRNA or ACL siRNA for 21 hours, followed by Western blot analysis as shown. (C and D) NIH 3 T3 fibroblasts (C) and human kidney fibroblasts (D) were stimulated with or without TCF- $\beta 1$ ( 5 ng/ $\mathrm{ml}$ ) for 3 hours and treated with $50 \mu \mathrm{M} \mathrm{ACL}$ inhibitor (ACLi) or DMSO (Control) for 21 hours, followed by Western blot analysis as shown. (E) NIH $3 T 3$ fibroblasts were treated with AKTi $(10 \mu \mathrm{M}$ or $20 \mu \mathrm{M})$, ACLi $(50 \mu \mathrm{M})$, or DMSO (Control) and stimulated with or without TCF- $\beta 1$ (5 ng/ml) for 24 hours. Cell proliferation was determined by MTS assay. Data are expressed as a percentage of the response compared with the cells treated with DMSO without TGF- $\beta 1$. Data were obtained from 4 wells per group. ${ }^{*} P<0.05$ compared with control with TGF- $\beta 1$ by ANOVA with Newman-Keuls post-hoc test. Values represent mean \pm SEM, with 3 independent experiments.

regulates necrosis-independent inflammation in dendritic cells and a tissue repair pathway in intestinal epithelium $(38,39)$. We hypothesized that RIPK3 may promote fibrosis under conditions when physiological tissue repair processes become excessive and maladaptive in the kidney. Moreover, RIPK3 can enhance the activity of 3 glucose metabolic enzymes, PYGL, GLUL, and GLUD1, in response to TNF- $\alpha$ (53). These observations suggest that the function of RIPK3 is not limited to the regulation of programmed cell death and may effect other cellular processes. Our data demonstrate that mice deficient in RIPK3 or its pseudokinase substrate MLKL displayed differential phenotypes in the UUO model of kidney fibrosis. In the current study, the levels of RIPK3 and MLKL expression, as well as p-MLKL, were induced in the obstructed kidney, but only mice deficient in RIPK3, and not mice deficient in MLKL, were protected against kidney fibrosis after UUO. Our results, taken together, suggest that RIPK3 contributes to the regulation of fibrogenesis in kidney tissue in a manner that is independent of MLKL. We cannot exclude the possibility that RIPK3-dependent, MLKL-independent necroptosis in fibroblasts, or other cell types such as tubular epithelial cells, may play a role in regulating kidney fibro-

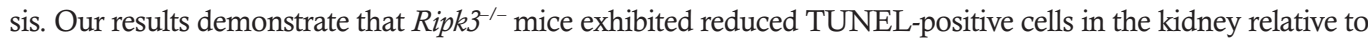
WT mice subjected to UUO. However, the absolute number of TUNEL-positive kidney cells was low in mice subjected to UUO and not detectable in mice subjected to AD. Fibroblast-specific and/or tubular epithelialspecific RIPK3 conditional knockout mice may be useful to address the role of RIPK3 in specific cell types.

In conclusion, we have identified a role of RIPK3 as a key regulator of TGF- $\beta$-induced fibrogenesis in vitro and kidney fibrosis in vivo, using two independent models of kidney fibrosis. Furthermore, we demonstrate that RIPK3 regulates fibrogenesis independently of MLKL by activating the AKT-dependent regulation of the metabolic enzyme ACL in fibroblasts. Our experiments suggest that drugs that inhibit ACL may have therapeutic benefit for patients with kidney fibrosis. Further studies will be required to determine the clinical usefulness of ACLi in CKD. 
A

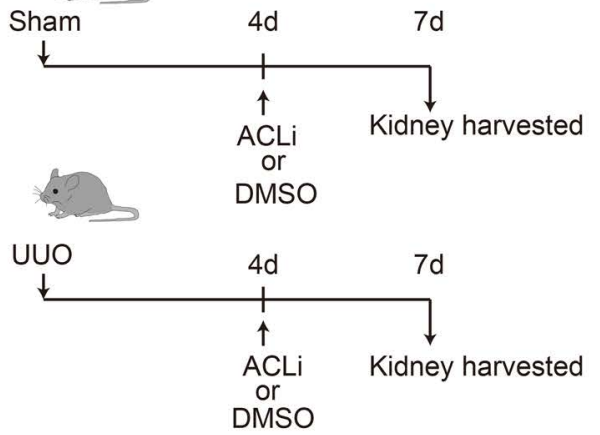

B
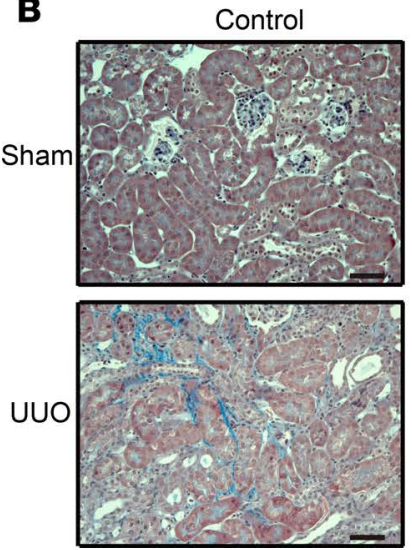
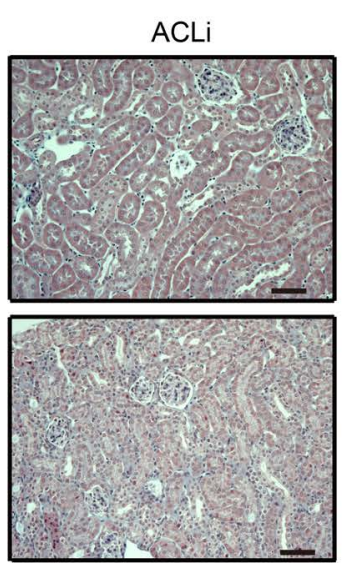

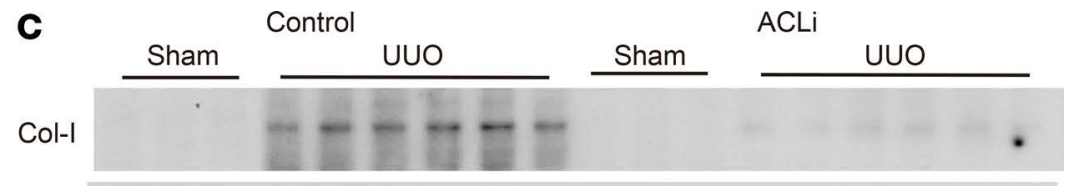

SMA

FN

GAPDH

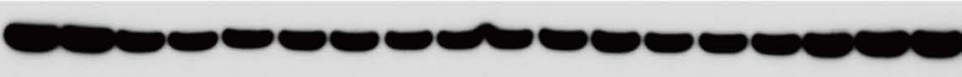

Figure 8. Inhibition of the ACL pathway attenuates UUO-induced kidney fibrosis in mice. (A) Scheme of the experimental approach. WT mice were subjected to sham operation or UUO. Four days after surgery, the mice were treated with ACL inhibitor (ACLi), SB 204990 (65 mg/kg, i.p.), or vehicle (DMSO, Control) and sacrificed 7 days after surgery. (B) Representative images of Masson's trichrome staining from kidney sections at day 7. (C) Western blotting analysis of kidney tissues of the sham-operated or UUO mice, which received vehicle or ACLi, at day 7. CAPDH was the standard. Dot plots represent densitometric analysis of Western blot data ( $n=3$ for Sham; $n=6$ for UUO). ${ }^{*} P<0.05$ compared with control UUO mice by ANOVA with Newman-Keuls post-hoc test. Values represent mean \pm SEM, with 3 independent experiments.

\section{Methods}

Mice. WT male C57BL/6 mice were purchased from Charles River. Ripk3 ${ }^{-1-}$ mice, which were backcrossed

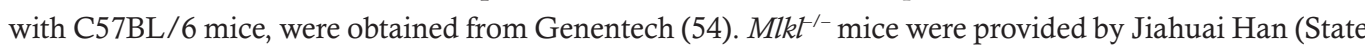
Key Laboratory of Cellular Stress Biology and School of Life Sciences, Xiamen University, Xiamen, China) (55). Eight- to twelve-week-old age- and sex-matched mice were used for experiments. Mice were maintained in the Weill Cornell Medicine pathogen-free facility.

UUO model of kidney fibrosis. UUO surgery was carried out as previously described (56). Male mice ( $n=2$ or 3 per group for sham, $n=5$ or 6 per group for UUO) were anesthetized, and ureteral obstruction was performed by the double ligation of the left ureter with 4-0 silk suture via a left flank incision. Sham-operated mice had their ureters exposed and manipulated but not ligated. Sham-operated kidneys were used as a control. The kidneys were collected at the end of the study for histology, Western blotting, and real-time PCR analysis.

AD-induced kidney fibrosis model. The $0.2 \% \mathrm{AD}$ (TD150071) and control diet were purchased from ENVIGO (32). The serum and kidneys were collected at the end of the study for the measurement of renal function, histology, and Western blotting.

Reagents. For Western blot analyses, antibodies against RIPK3 (PRS2283), MLKL (SAB1302339), and $\beta$-actin (A2228) were from MilliporeSigma. Antibodies to AKT (C67E7), p-AKT (D9E, Ser473), ACL (4332), p-ACL (4331, Ser455), and FASN (C20G5) were from Cell Signaling Technology. Antibodies to human RIPK3 (2B10), Col-1 (ab21286), a-SMA (ab5694), FN (ab2413), and p-MLKL (ab196436) were from Abcam. Antibody to GAPDH (FL335) was from Santa Cruz. RIPK3 inhibitor (GSK'872) was from Calbiochem. AKTi (A6730) and Nec-1 (N9037) were from MilliporeSigma. ACLi (SB 204990) was from Tocris Bioscience. 
A
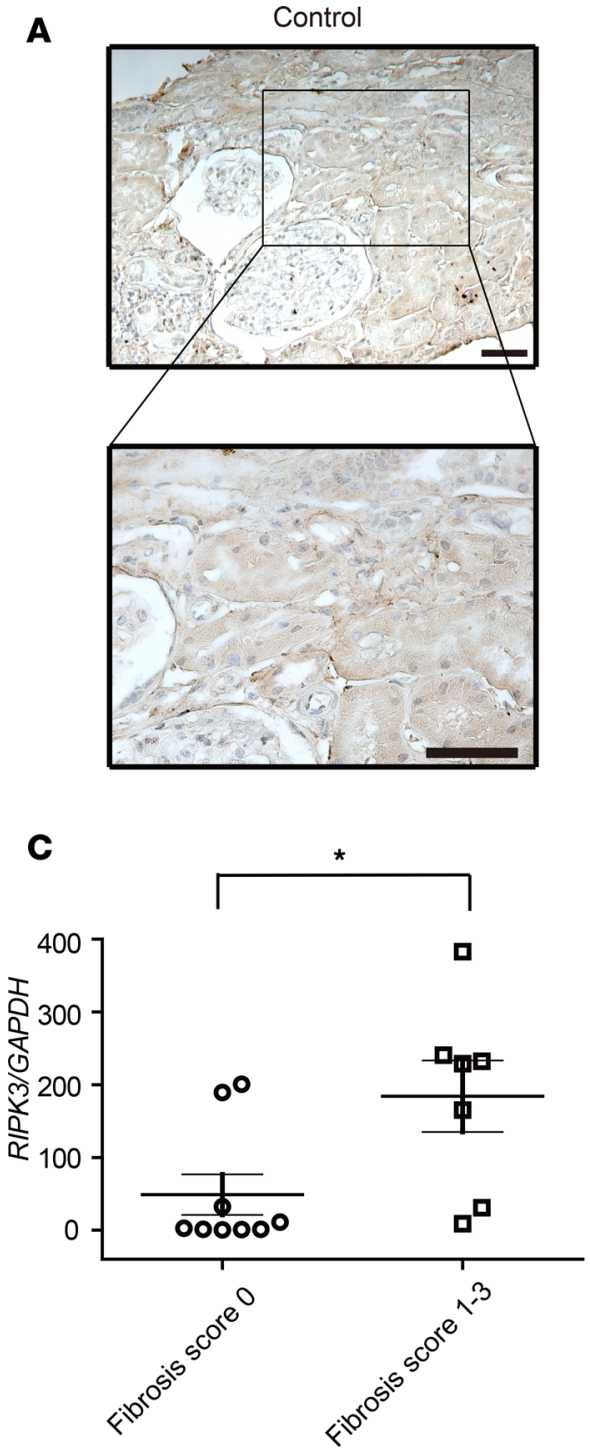

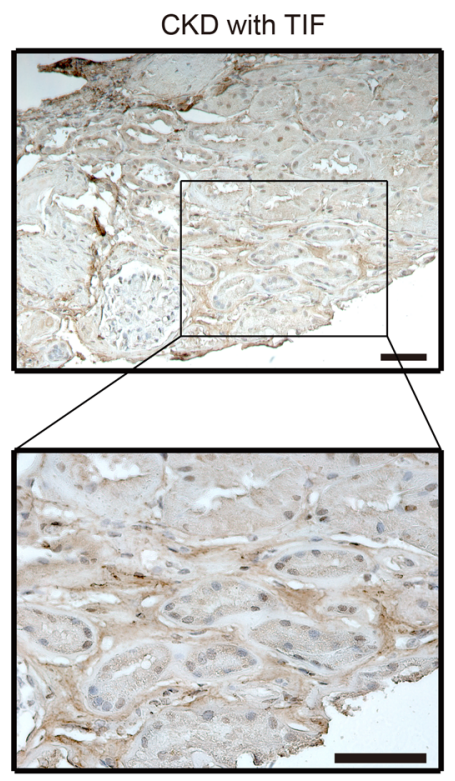

D

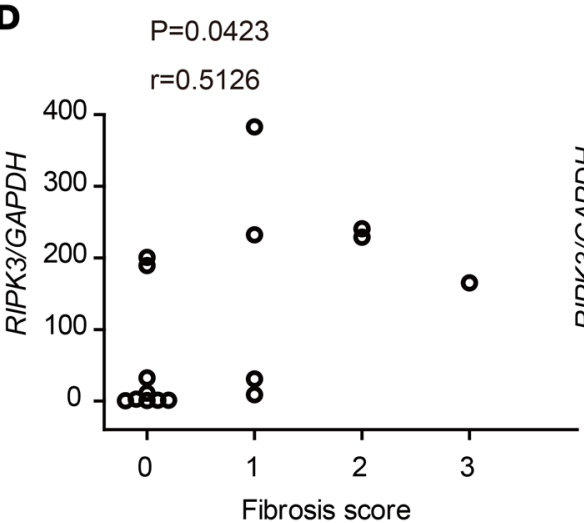

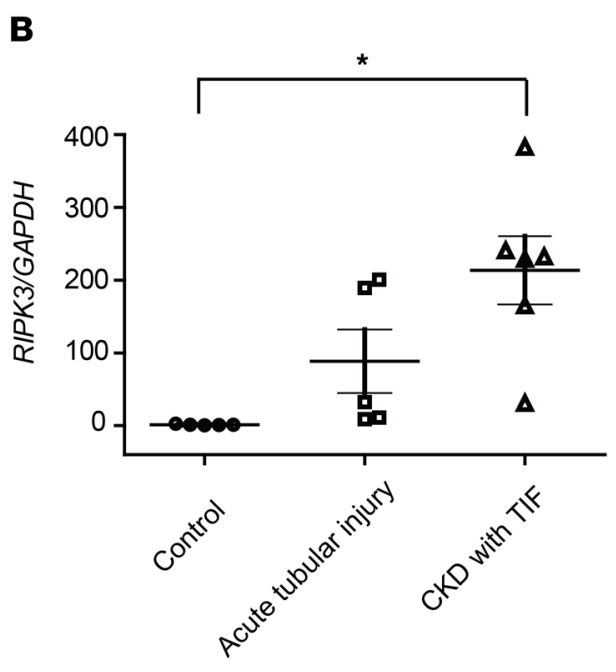

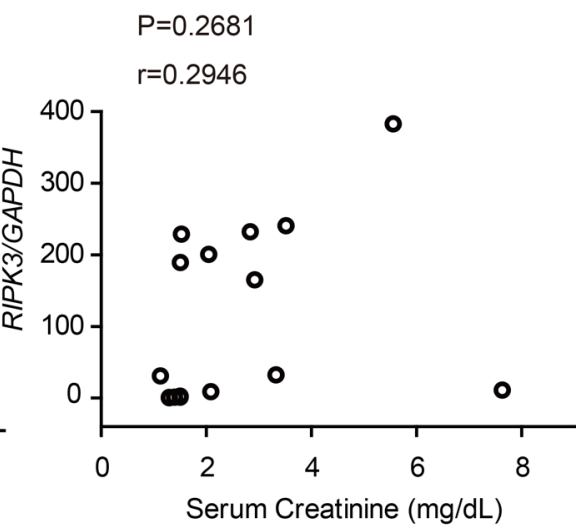

Figure 9. RIPK3 expression in human kidney disease. (A) Representative immunohistochemistry for RIPK3 protein expression in human kidney allograft sections from CKD patients with diabetic nephropathy and histological evidence of TIF or control subjects without diabetic nephropathy. Scale bars: $100 \mu \mathrm{m}$. (B) Relative levels of RIPK3/GAPDH mRNA expression were determined by qRT-PCR in kidney biopsy samples from CKD patients with diabetic nephropathy and TIF $(n=6)$ compared with patients with acute tubular injury $(n=5)$ and control patient samples of normal kidney tissue margins from tumor resection $(n=5) .{ }^{*} P<0.01$. Error bars represent mean \pm SEM. (C) Relative levels of RIPK3/GAPDH mRNA expression in kidneys with fibrosis score $=0$ and fibrosis score $=1-3 .{ }^{*} P<0.05$. Error bars represent mean \pm SEM. (D) Correlation between RIPK3/GAPDH mRNA levels in kidney tissues and interstitial fibrosis score and serum creatinine. Data were calculated using ANOVA with Newman-Keuls post-hoc test (B), Mann-Whitney test (C), and Pearson's correlation (D).

Genotyping. The genotype of each mouse was determined by the PCR from genomic DNA obtained from tail biopsies. The following primer sequences were used for the genotyping: MLKL forward, AGCCCAAAGAGGCAGCACAAATC; MLKL reverse, AAACTTCCAAATATGGGACTTCTTG.

Western blot analysis. Kidney tissues were lysed with a buffer containing Tissue Extraction Reagent (Invitrogen), a protease inhibitor cocktail (Roche), and phosphatase inhibitors (MilliporeSigma). Cells were lysed with NP-40 Lysis Buffer (FNN0021, Invitrogen), a protease inhibitor cocktail, and phosphatase inhibitors. Lysates were centrifuged for 10 minutes at $4^{\circ} \mathrm{C}$, and the supernatants were obtained. The protein concentrations of the supernatants were determined using the Bradford assay (Bio-Rad Laboratories). Proteins were subjected to NuPAGE 4\%-12\% Bis-Tris gels (Invitrogen) and transferred to Protran nitrocellulose membranes (GE Healthcare Life Science).

qRT-PCR analysis. Total RNA was isolated from the kidney using TRIzol (Invitrogen). $2 \mu \mathrm{g}$ RNA was reverse transcribed using the High-Capacity DNA Reverse Transcription kit (Applied Biosystems), and qRT-PCR was performed in the ABI PRISM 7500 Real-Time PCR System (Applied Biosystems) using 
SYBRGreen Master Mix and gene-specific primers. The data were normalized by the ratio to Gapdh. The primers used are listed in Supplemental Table 2.

Histology and immunostaining. We used formalin-fixed, paraffin-embedded kidney sections stained with Masson's trichrome. Immunohistochemical analysis was performed using specific primary antibodies (antibody to mouse RIPK3 [Cell Signaling, 15825], antibody to human RIPK3 [Abcam, 2B10]) and biotinylated secondary antibodies (Vector Laboratories).

In a separate experiment, immunofluorescent staining was performed using cryosections $(15 \mu \mathrm{m})$. Primary antibody against mouse RIPK3 (Cell Signaling, 15825) and antibody against Vimentin (abcam, 24525) were used without antigen retrieval on cryosections. Staining was detected by Alexa Fluor 488 conjugated (green, Molecular Probes) or Alexa Fluor 555-conjugated (red, Molecular Probes) anti-IgG as secondary antibody. Nuclei were stained with Hoechst 33342 (Thermofisher Scientific, 62249).

For TUNEL staining, formalin-fixed, paraffin-embedded kidney sections were stained using the ApoAlert DNA Fragmentation Assay Kit (Clontech), according to the manufacturer's protocol. Slides were examined using a LSM880 confocal microscope with Airyscan High Resolution Detector. Both DAPI- and TdT-positive cells subjected to nuclear staining were counted as TUNEL-positive cells. The number of TUNEL-positive cells was counted in 5 random high-power fields (HPF) $(1 \mathrm{HPF}=637 \mu \mathrm{m} \times 637 \mu \mathrm{m})$.

Cell culture. NIH 3T3 fibroblasts were obtained from ATCC. NIH 3T3 cells were maintained in ATCC complete medium (DMEM supplemented with 10\% bovine calf serum, 1\% penicillin-streptomycin) at $37^{\circ} \mathrm{C}$ with $5 \% \mathrm{CO}_{2}$. Human kidney fibroblasts (AU009) were obtained from dv biologics. Human kidney fibroblasts were maintained in fibroblast cellutions medium (I-GRO-001-500, dv biologics). rTGF- $\beta 1$ (R\&D systems) was used to stimulate cells in vitro. Briefly, cells were plated at $1 \times 10^{5}$ to $2 \times 10^{5}$ cells/well in a 6-well plate and allowed to attach for 12-24 hours. Then, the medium was changed to OPTI-MEM (GIBCO) supplemented with $1 \%$ bovine calf serum 1 hour prior to TGF- $\beta 1$ treatment $(5 \mathrm{ng} / \mathrm{ml}$ or $0.1-10$ $\mathrm{ng} / \mathrm{ml}$, as indicated in Figure $5 \mathrm{~A}$, or $5 \mathrm{ng} / \mathrm{ml}$ or $10 \mathrm{ng} / \mathrm{ml}$, as indicated in Figure $5 \mathrm{C}$ ). For chemical inhibition of RIPK3 or RIPK1, cells were treated with $10 \mu \mathrm{M}$ GSK'872 (Calbiochem) or $60 \mu \mathrm{M}$ Nec-1 for 1 hour and then treated with TGF- $\beta 1(5 \mathrm{ng} / \mathrm{ml})$ for 24 hours. For chemical inhibition of AKT, cells were treated with AKTi $(10 \mu \mathrm{M})$ and TGF- $\beta 1(5 \mathrm{ng} / \mathrm{ml})$ at the same time and incubated for 24 hours. For chemical inhibition of ACL, cells were treated with TGF- $\beta 1(5 \mathrm{ng} / \mathrm{ml})$ for 3 hours and then treated with $50 \mu \mathrm{M}$ ACLi (Tocris) for 21 hours.

Transfection of siRNA. For knockdown of mouse RIPK3, 1 mixture of siRNA (Dharmacon, SMART Pool, L-049919-00-0005) and 3 independent siRNAs (Dharmacon, ON-TARGET plus, J-049919-05-0005, J-049919-06-0005, and J-049919-07-0005) were used. Mouse AKT1 siRNA (Dharmacon, SMART Pool, L-040709-00-0005), mouse ACL siRNA (MilliporeSigma, EMU005061), human AKT1 siRNA (MilliporeSigma, EHU083501), and human ACL siRNA (MilliporeSigma, EHU081921) were also used for knockdown of each gene. NIH 3T3 fibroblasts or human kidney fibroblasts $\left(1 \times 10^{5}\right.$ to $2 \times 10^{5}$ cells per well) were seeded in 6-well plates and were transfected with siRNA for target molecules or siRNA negative control (Dharmacon, ON-TARGET plus Non-targeting Pool, D-001810-10-05) using Lipofectamine RNAiMAX Transfection Unit (13778-075, Invitrogen) according to the manufacturer's instructions (16, 17).

MTS assay. $1 \times 10^{4} \mathrm{NIH} 3 \mathrm{~T} 3$ fibroblasts were seeded in 96-well plates overnight and incubated with ACLi $(50 \mu \mathrm{M})$, AKTi $(10 \mu \mathrm{M}$ or $20 \mu \mathrm{M})$, or with appropriate vehicle controls. Cells were stimulated with TGF- $\beta$ ( $5 \mathrm{ng} / \mathrm{ml}$ ) for 24 hours. Cell proliferation was assessed by MTS (tetrazolium compound [3-(4,5-dimethylthiazol-2-yl)-5-(3-carboxymethoxyphenyl)-2-(4-sulfophenyl)-2H-tetrazolium, inner salt) assay according to the manufacturer's instructions (CellTiter 96 AQueous One Solution Cell Proliferation Assay, Promega).

Human kidney samples. We analyzed human kidney specimens obtained from allograft biopsies without evidence of rejection (6 with diabetic nephropathy and tubular interstitial fibrosis, 5 with acute tubular injury) and normal kidney specimens $(n=5)$ from native nephrectomies for renal carcinoma (Supplemental Table 1). Interstitial fibrosis was determined by Masson's trichrome stain and was scored by the pathologist based on the Banff classification of renal allograft pathology. Quantitative criteria for interstitial fibrosis (Banff ci score) are as follows: ci0, interstitial fibrosis in up to $5 \%$ of cortical area; ci1-mild, interstitial fibrosis in 6\%-25\% of cortical area; ci2-moderate, interstitial fibrosis in $26 \%-50 \%$ of cortical area; and ci3-severe, interstitial fibrosis in $>50 \%$ of cortical area (57). All biopsies were performed for clinical indications at Weill Cornell Medical Center or Memorial Sloan Kettering Cancer Center.

Statistics. All statistical tests were analyzed with statistical software (GraphPad Prism version 4.0). Statistical analysis was performed using 1-way ANOVA followed by Newman-Keuls post-hoc test. Mann-Whitney 
test was used to compare two groups. Pearson's correlation analysis was used in Figure 9D. $P$ values of less than 0.05 were considered statistically significant.

Study approval. All animal experiments were performed under animal study protocols approved by the Institutional Animal Care and Use Committee of Weill Cornell Medicine. The study protocol was approved by the Human Subjects Review Committee of Weill Cornell Medicine and Memorial Sloan Kettering Cancer Center, and written informed consent was obtained from all human subjects.

\section{Author contributions}

MI, KN, AMKC, and MEC conceived the study; MI performed the majority of experiments. JSM and KPC contributed to experiments. TM and RS contributed human clinical samples and analysis; MI, SWR, AMKC, and MEC wrote and edited the paper; and AMKC and MEC supervised the entire project.

\section{Acknowledgments}

We thank B. He (Weill Cornell Medical College) for help with immunohistochemistry and Y. Ding (Weill Cornell Medical College), I. Siempos (Weill Cornell Medical College), E. Patino (Weill Cornell Medical College), and S. Angara (Weill Cornell Medical College) for technical assistance. This work was supported in part by NIH grants R01 DK57661 to MEC and R01 HL060234 and R01 HL133801 to MEC and AMKC.

Address correspondence to: Mary E. Choi, Division of Nephrology and Hypertension, Joan and Sanford I. Weill Department of Medicine, Weill Cornell Medical College, 525 East 68th Street, Box 3, New York, New York 10065, USA. Phone: 646.962.2605; Email: mechoi@med.cornell.edu. Or to: Augustine M. K. Choi, Division of Pulmonary and Critical Care Medicine, Joan and Sanford I. Weill Department of Medicine, Weill Cornell Medical College, 1300 York Avenue, Suite F-113 Box 83, New York, New York 10065, USA. Phone: 212.746.5900; Email: amc2056@med.cornell.edu.

1. Chawla LS, Eggers PW, Star RA, Kimmel PL. Acute kidney injury and chronic kidney disease as interconnected syndromes. N Engl J Med. 2014;371(1):58-66.

2. Klahr S, Morrissey J. Obstructive nephropathy and renal fibrosis. Am J Physiol Renal Physiol. 2002;283(5):F861-F875.

3. Rockey DC, Bell PD, Hill JA. Fibrosis--A common pathway to organ injury and failure. N Engl J Med. 2015;373(1):96.

4. Wynn TA, Ramalingam TR. Mechanisms of fibrosis: therapeutic translation for fibrotic disease. Nat Med. 2012;18(7):1028-1040.

5. Liu Y. Renal fibrosis: new insights into the pathogenesis and therapeutics. Kidney Int. 2006;69(2):213-217.

6. Meng XM, Tang PM, Li J, Lan HY. TGF- $\beta$ /Smad signaling in renal fibrosis. Front Physiol. 2015;6:82.

7. Choi ME, Ding Y, Kim SI. TGF- $\beta$ signaling via TAK1 pathway: role in kidney fibrosis. Semin Nephrol. 2012;32(3):244-252.

8. Hawinkels LJ, Ten Dijke P. Exploring anti-TGF- $\beta$ therapies in cancer and fibrosis. Growth Factors. 2011;29(4):140-152

9. Lee SY, Kim SI, Choi ME. Therapeutic targets for treating fibrotic kidney diseases. Transl Res. 2015;165(4):512-530.

10. Horowitz JC, et al. Activation of the pro-survival phosphatidylinositol 3-kinase/AKT pathway by transforming growth factor-beta 1 in mesenchymal cells is mediated by p38 MAPK-dependent induction of an autocrine growth factor. J Biol Chem. 2004;279(2):1359-1367.

11. Manning BD, Cantley LC. AKT/PKB signaling: navigating downstream. Cell. 2007;129(7):1261-1274.

12. Cantley LC. The phosphoinositide 3-kinase pathway. Science. 2002;296(5573):1655-1657.

13. Rodríguez-Peña AB, et al. Activation of Erk1/2 and Akt following unilateral ureteral obstruction. Kidney Int. 2008;74(2):196-209.

14. Berwick DC, Hers I, Heesom KJ, Moule SK, Tavare JM. The identification of ATP-citrate lyase as a protein kinase B (Akt) substrate in primary adipocytes. J Biol Chem. 2002;277(37):33895-33900.

15. Zaidi N, Swinnen JV, Smans K. ATP-citrate lyase: a key player in cancer metabolism. Cancer Res. 2012;72(15):3709-3714.

16. Moon JS, et al. UCP2-induced fatty acid synthase promotes NLRP3 inflammasome activation during sepsis. J Clin Invest. 2015;125(2):665-680.

17. Moon JS, et al. mTORC1-Induced HK1-dependent glycolysis regulates NLRP3 inflammasome activation. Cell Rep. 2015;12(1):102-115.

18. Ghesquière B, Wong BW, Kuchnio A, Carmeliet P. Metabolism of stromal and immune cells in health and disease. Nature. 2014;511(7508):167-176.

19. Liu H, et al. Mass spectrometry imaging of kidney tissue sections of rat subjected to unilateral ureteral obstruction. Sci Rep. 2017;7:41954

20. Kang HM, et al. Defective fatty acid oxidation in renal tubular epithelial cells has a key role in kidney fibrosis development. Nat Med. 2015;21(1):37-46.

21. Mizumura K, et al. Mitophagy-dependent necroptosis contributes to the pathogenesis of COPD. J Clin Invest. 2014;124(9):3987-4003.

22. Pasparakis M, Vandenabeele P. Necroptosis and its role in inflammation. Nature. 2015;517(7534):311-320.

23. Vitner EB, et al. RIPK3 as a potential therapeutic target for Gaucher's disease. Nat Med. 2014;20(2):204-208. 
24. Cho YS, et al. Phosphorylation-driven assembly of the RIP1-RIP3 complex regulates programmed necrosis and virus-induced inflammation. Cell. 2009;137(6):1112-1123.

25. Sun L, et al. Mixed lineage kinase domain-like protein mediates necrosis signaling downstream of RIP3 kinase. Cell. 2012;148(1-2):213-227.

26. Rickard JA, et al. RIPK1 regulates RIPK3-MLKL-driven systemic inflammation and emergency hematopoiesis. Cell. 2014;157(5):1175-1188.

27. Linkermann A, et al. Rip1 (receptor-interacting protein kinase 1) mediates necroptosis and contributes to renal ischemia/reperfusion injury. Kidney Int. 2012;81(8):751-761.

28. Linkermann A, et al. Two independent pathways of regulated necrosis mediate ischemia-reperfusion injury. Proc Natl Acad Sci USA. 2013;110(29):12024-12029.

29. Linkermann A, et al. The RIP1-kinase inhibitor necrostatin-1 prevents osmotic nephrosis and contrast-induced AKI in mice. $J$ Am Soc Nephrol. 2013;24(10):1545-1557.

30. Wang S, Zhang C, Hu L, Yang C. Necroptosis in acute kidney injury: a shedding light. Cell Death Dis. 2016;7:e2125.

31. Gautheron J, et al. A positive feedback loop between RIP3 and JNK controls non-alcoholic steatohepatitis. EMBO Mol Med 2014;6(8):1062-1074.

32. Akchurin $\mathrm{O}$, et al. Lack of hepcidin ameliorates anemia and improves growth in an adenine-induced mouse model of chronic kidney disease. Am J Physiol Renal Physiol. 2016;311(5):F877-F889.

33. Wu XN, et al. Distinct roles of RIP1-RIP3 hetero- and RIP3-RIP3 homo-interaction in mediating necroptosis. Cell Death Differ 2014;21(11):1709-1720

34. Wang X, et al. RNA viruses promote activation of the NLRP3 inflammasome through a RIP1-RIP3-DRP1 signaling pathway. Nat Immunol. 2014;15(12):1126-1133.

35. Moriwaki K, Balaji S, McQuade T, Malhotra N, Kang J, Chan FK. The necroptosis adaptor RIPK3 promotes injury-induced cytokine expression and tissue repair. Immunity. 2014;41(4):567-578.

36. Chan FK, Luz NF, Moriwaki K. Programmed necrosis in the cross talk of cell death and inflammation. Annu Rev Immunol. 2015;33:79-106

37. Lawlor KE, et al. RIPK3 promotes cell death and NLRP3 inflammasome activation in the absence of MLKL. Nat Commun. 2015;6:6282.

38. Martin-Sanchez D, et al. Ferroptosis, but not necroptosis, is important in nephrotoxic folic acid-induced AKI. J Am Soc Nephrol. 2017;28(1):218-229.

39. Newton K, et al. RIPK3 deficiency or catalytically inactive RIPK1 provides greater benefit than MLKL deficiency in mouse models of inflammation and tissue injury. Cell Death Differ. 2016;23(9):1565-1576.

40. Borges FT, et al. TGF- $\beta 1$-containing exosomes from injured epithelial cells activate fibroblasts to initiate tissue regenerative responses and fibrosis. J Am Soc Nephrol. 2013;24(3):385-392.

41. Mandal P, et al. RIP3 induces apoptosis independent of pronecrotic kinase activity. Mol Cell. 2014;56(4):481-495

42. Kaiser WJ, et al. RIP1 suppresses innate immune necrotic as well as apoptotic cell death during mammalian parturition. Proc Natl Acad Sci USA. 2014;111(21):7753-7758.

43. Varga J, Pasche B. Transforming growth factor beta as a therapeutic target in systemic sclerosis. Nat Rev Rheumatol. 2009;5(4):200-206.

44. Laplante M, Sabatini DM. mTOR signaling in growth control and disease. Cell. 2012;149(2):274-293.

45. Shaw RJ, Cantley LC. Ras, PI(3)K and mTOR signalling controls tumour cell growth. Nature. 2006;441(7092):424-430.

46. Hatzivassiliou G, et al. ATP citrate lyase inhibition can suppress tumor cell growth. Cancer Cell. 2005;8(4):311-321.

47. Hanai JI, Doro N, Seth P, Sukhatme VP. ATP citrate lyase knockdown impacts cancer stem cells in vitro. Cell Death Dis. 2013;4:e696.

48. Xiao X, Du C, Yan Z, Shi Y, Duan H, Ren Y. Inhibition of necroptosis attenuates kidney inflammation and interstitial fibrosis induced by unilateral ureteral obstruction. Am J Nephrol. 2017;46(2):131-138.

49. Li Q, et al. Receptor interacting protein 3 suppresses vascular smooth muscle cell growth by inhibition of the phosphoinositide 3-kinase-Akt axis. J Biol Chem. 2010;285(13):9535-9544.

50. Canaud G, et al. AKT2 is essential to maintain podocyte viability and function during chronic kidney disease. Nat Med. 2013;19(10):1288-1296.

51. Lan A, Zhang J, Xiao Z, Peng X, Qi Y, Du J. Akt2 is involved in loss of epithelial cells and renal fibrosis following unilateral ureteral obstruction. PLoS One. 2014;9(8):e105451.

52. Zhang T, et al. CaMKII is a RIP3 substrate mediating ischemia- and oxidative stress-induced myocardial necroptosis. Nat Med 2016;22(2):175-182.

53. Zhang DW, et al. RIP3, an energy metabolism regulator that switches TNF-induced cell death from apoptosis to necrosis. Science. 2009;325(5938):332-336.

54. Newton K, Sun X, Dixit VM. Kinase RIP3 is dispensable for normal NF-kappa Bs, signaling by the B-cell and T-cell receptors, tumor necrosis factor receptor 1, and Toll-like receptors 2 and 4. Mol Cell Biol. 2004;24(4):1464-1469.

55. Wu J, et al. Mlkl knockout mice demonstrate the indispensable role of Mlkl in necroptosis. Cell Res. 2013;23(8):994-1006.

56. Ding Y, Kim S1, Lee SY, Koo JK, Wang Z, Choi ME. Autophagy regulates TGF- $\beta$ expression and suppresses kidney fibrosis induced by unilateral ureteral obstruction. J Am Soc Nephrol. 2014;25(12):2835-2846.

57. Racusen LC, et al. The Banff 97 working classification of renal allograft pathology. Kidney Int. 1999;55(2):713-723. 